\title{
EXPONENTIATION IN BANACH STAR ALGEBRAS
}

\author{
by H. G. DALES $\dagger$ \\ (Received 28th January 1975)
}

In their recently published book Bonsall and Duncan ask the following question ((1), p. 65): if $A$ is a Banach star algebra with an identity, is it true that

$$
\exp \left(a^{*}\right)=(\exp a)^{*} \quad(a \in A) ?
$$

Of course, if the involution * is continuous, this result does hold. However, in this note, we record a counter-example. In fact, the example is a slight modification of a result of Loy (3), in turn based on a construction in (2), which demonstrates the possible non-uniqueness of the exponential of an element in a commutative Banach algebra with two inequivalent complete norms.

Proposition 1. There exists a commutative Banach star algebra $B$ with identity and an element $b \in B$ for which $\exp \left(b^{*}\right) \neq(\exp b)^{*}$.

Proof. Let $A$ denote the disc algebra, let $X$ denote the Banach space $C[0,1]$ (with the usual notation), and let $T \in B(X)$ be the Volterra operator defined by

$$
(T x)(t)=\int_{0}^{t} x(s) d s \quad(x \in X) .
$$

Then, with respect to the operation $(f, x) \rightarrow f \cdot x=f(T) x, A \times X \rightarrow X, X$ is a commutative Banach $A$-module (cf. (2)). Now let $\mathfrak{U}=A \oplus X$, let

$$
\left(a_{1}, x_{1}\right)\left(a_{2}, x_{2}\right)=\left(a_{1} a_{2}, a_{1} \cdot x_{2}+a_{2} \cdot x_{1}\right) \text {, }
$$

and let $\|(a, x)\|_{1}=\|a\|+\|x\|$. Then $\mathfrak{U}_{1}=\left(\mathfrak{U},\|\cdot\|_{1}\right)$ is a commutative Banach algebra with identity.

Let $*$ be the standard involution on $A$ defined by $f^{*}(z)=\overline{f(\bar{z})}(f \in A)$, where ${ }^{-}$denotes complex-conjugation, and define $*: \mathfrak{U} \rightarrow \mathfrak{A}$ by

$$
(f, x)^{*}=\left(f^{*}, \bar{x}\right) \quad((f, x) \in \mathfrak{U}) .
$$

To check that $*$ is indeed an involution on $\mathfrak{A}$, it suffices to check that

$$
\overline{(f \cdot x)}=f^{*} \cdot \bar{x} \quad(f \in A, x \in X) \text {. }
$$

But, if $f(z)=\sum_{0}^{\infty} \lambda_{n} z^{n}$, so that $f^{*}(z)=\sum_{0}^{\infty} \bar{\lambda}_{n} z^{n}$,

$$
f^{*} \cdot \bar{x}=\sum_{0}^{\infty} \bar{\lambda}_{n} T^{n} \bar{x}=\sum_{0}^{\infty} \lambda_{n} \overline{\left(T^{n} x\right)}=\overline{(f \cdot x)}
$$

as required. Thus $\left(\mathfrak{U}_{1}, *\right)$ is a Banach star algebra.

† Supported in part by NSF grant GP-33431. 
Let $D: A \rightarrow X$ be a discontinuous derivation with $D(p)=0$ for $p$ a polynomial, but with $D(E)=x_{0} \neq 0$, where $E(z)=e^{z}$. Such a derivation is constructed in (2). Define

$$
\|(f, x)\|_{2}=\|f\|+\|D f-x\| \quad((f, x) \in \mathfrak{2}) .
$$

(This norm is introduced in (3).) It is clear that $\|.\|_{2}$ is an algebra norm on $\mathfrak{U}$. We check directly that it is complete. Let $\left(\left(f_{n}, x_{n}\right)\right)$ be a $\|\cdot\|_{2}$-Cauchy sequence, so that $\left(f_{n}\right)$ is Cauchy in $A$ and $\left(D f_{n}-x_{n}\right)$ is Cauchy in $X$. Let $f_{n} \rightarrow f \in A$ and let $D f_{n}-x_{n} \rightarrow y \in X$. Then it is clear that

$$
\left(f_{n}, x_{n}\right) \rightarrow(f, D f-y)
$$

with respect to $\|\cdot\|_{2}$. Thus, $\mathfrak{A}_{2}=\left(\mathfrak{A},\|\cdot\|_{2}\right)$ is a commutative Banach algebra.

Let $B$ denote the direct sum $\mathfrak{A}_{1} \oplus \mathfrak{A}_{2}$ with the norm

with the product

$$
\|(a, b)\|=\max \left\{\|a\|_{1},\|b\|_{2}\right\},
$$

and the involution

$$
\left(a_{1}, b_{1}\right)\left(a_{2}, b_{2}\right)=\left(a_{1} a_{2}, b_{1} b_{2}\right),
$$

$$
(a, b)^{*}=\left(b^{*}, a^{*}\right) \text {. }
$$

Then $B$ is a commutative Banach star algebra with a discontinuous involution ((1), Example 36.11).

Let $a=(z, 0) \in \mathfrak{A}$, where $z$ denotes the coordinate functional in $A$, and let $\exp _{i} a$ denote the exponential of $a$ in $\mathfrak{A}_{i}(i=1,2)$. Precisely,

$$
\exp _{i} a=\lim _{\|\cdot\|_{i}} \sum_{k=0}^{n} \frac{a^{k}}{k !} \text { as } n \rightarrow \infty \text {. }
$$

Then $\exp _{1} a=(E, 0)$, but, by (2), $\exp _{2} a=\left(E, x_{0}\right) \neq \exp _{1} a$. Finally, let $b=(a, 0) \in B$. Then, noting that $\boldsymbol{a}$ is self-adjoint in $\mathfrak{A}$,

whereas

$$
\exp \left(b^{*}\right)=\exp \left(0, a^{*}\right)=\exp (0, a)=\left(0, \exp _{2} a\right),
$$

$$
(\exp b)^{*}=(\exp (a, 0))^{*}=\left(\exp _{1} a, 0\right)^{*}=\left(0, \exp _{1} a\right),
$$

so that $\exp \left(b^{*}\right) \neq(\exp b)^{*}$, and we have constructed the required example.

Remark. By taking $h=(a, a) \in B$ we obtain an Hermitian element $h$ in $B$ for which $\exp h \neq(\exp h)^{*}$, so answering a question raised by B. E. Johnson in the review of (3), Math. Reviews 50 \#2918.

We do have a positive result in a special case. We say that a commutative Banach algebra $A$ has a unique functional calculus if, for each $a \in A$, there is a unique unital algebra homomorphism $\Theta_{a}: \mathcal{O}_{\sigma(a)} \rightarrow A$ such that $\Theta_{a}(z)=a$. Here $\sigma(a)$ denotes the spectrum of $a$ in $\mathbb{C}$, and $\mathcal{O}_{\sigma(a)}$ denotes the algebra of analytic functions on $\sigma(a)$ (cf. (2)). 
Proposition 2. Let $(A, *)$ be a commutative Banach algebra with a unique functional calculus. Then $\exp \left(a^{*}\right)=(\exp a)^{*}(a \in A)$.

Proof. Let $a \in A$. First note that $\sigma\left(a^{*}\right)=\overline{\sigma(a)}$, so that, if $F \in \mathcal{O}_{\sigma(a)}$, then $F^{*} \in \mathcal{O}_{\sigma\left(a^{*}\right)}$, where $F^{*}(z)=\overline{F(\bar{z})}$. Define $\Theta_{a}^{*}: \mathcal{O}_{\sigma(a)} \rightarrow A$ by

$$
\Theta_{a}^{*}(F)=\left[\Theta_{a^{*}}\left(F^{*}\right)\right]^{*} \quad\left(F \in \mathcal{O}_{\sigma(a)}\right)
$$

Then $\Theta_{a}^{*}$ is clearly a unital algebra homomorphism, and

$$
\Theta_{a}^{*}(z)=\left[\Theta_{a^{*}}(z)\right]^{*}=\left(a^{*}\right)^{*}=a,
$$

so that, by the uniqueness of the functional calculus $\Theta_{a}^{*}=\Theta_{a}$. In particular, $\left(\exp \left(a^{*}\right)\right)^{*}=\exp a$, as required.

Note that a Banach algebra with a finite-dimensional radical has a unique functional calculus (2). It is easy to give examples of such algebras which have a discontinuous involution.

\section{REFERENCES}

(1) F. F. Bonsall and J. Duncan, Complete normed algebras (Springer-Verlag, 1973).

(2) H. G. Dales, The uniqueness of the functional calculus, Proc. London Math. Soc. 27 (1973), 638-648.

(3) R. J. LoY, Commutative Banach algebras with non-unique complete norm topology, Bull. Austral. Math. Soc. 10 (1974), 409-420.

SCHOOL OF Mathematics

UNIVERSITY OF LEEDS

LEEDS LS2 9JT 